\title{
Le contrôle du temps de travail
}

\author{
María Rosa Vallecillo Gámez
}

\section{(2) OpenEdition}

Journals

Édition électronique

URL : https://journals.openedition.org/rdctss/1257

DOI : $10.4000 /$ rdctss. 1257

ISSN : 2262-9815

Éditeur

Centre de droit comparé du travail et de la sécurité sociale

Édition imprimée

Date de publication : 1 avril 2020

Pagination : 176-179

ISSN : 2117-4350

\section{Référence électronique}

María Rosa Vallecillo Gámez, "Le contrôle du temps de travail », Revue de droit comparé du travail et de la sécurité sociale [En ligne], 1 | 2020, mis en ligne le 01 novembre 2021, consulté le 11 novembre 2021 URL : http://journals.openedition.org/rdctss/1257 ; DOI : https://doi.org/10.4000/rdctss. 1257

\section{(c) (i) (9)}

Revue de droit comparé du travail et de la sécurité sociale est mise à disposition selon les termes de la Licence Creative Commons Attribution - Pas d'Utilisation Commerciale - Pas de Modification 4.0 International. 
MARÍA ROSA VALLECILLO GÁMEZ

UNIVERSITÉ DE JAÉN

\section{LE CONTRÔLE DU TEMPS DE TRAVAIL}

\section{I - LA NOUVELLE RÉGLEMENTATION SUR LA TENUE D'UN REGISTRE DU TEMPS DE TRAVAIL}

L'article 34 du Statut des travailleurs a été modifié le 12 mai 2019 par le Décret-loi royal n8/2019 du 8 mars 2019 relatif aux mesures urgentes de protection sociale et de lutte contre la précarité. Les entreprises espagnoles doivent désormais tenir un registre du temps de travail journalier de leurs salariés, indiquant les horaires de début et de fin de la journée de travail. Ce registre est obligatoire pour l'ensemble des salariés, quel que soit le secteur d'activité, et concerne également le télétravail et d'autres formes de travail flexibles. Seules certaines catégories ne sont pas visées (comme les cadres dirigeants des entreprises).

Le contrôle de la journée de travail n'a pas fait l'objet d'une réglementation satisfaisante sur le plan juridique. L'exigence d'un enregistrement du temps de travail journalier, limité aux heures supplémentaires effectuées par les travailleurs, n'a pas permis d'apporter une meilleure protection à ces derniers, pas plus qu'il n'a constitué un moyen objectif de prouver le respect des limites légales de l'aménagement du temps de travail.

Face à la polémique judiciaire qui résulte des divergences de vues entre l'Audiencia Nacional (Cour centrale) et le Tribunal Supremo (Cour suprême), la Cour de Justice de I'Union Européenne (CJUE) saisie d'une demande de décision préjudicielle s'est prononcée le 14 mai 2019 (affaire C-55/18), quelques jours seulement après la publication de la réglementation sur le registre du temps de travail.

L'arrêt de la Cour considère qu'au vu des articles 3, 5 et 6 de la Directive 2003/88, les États membres doivent imposer aux employeurs l'obligation de mettre en place un système permettant de mesurer la durée du temps de travail journalier effectué par chaque salarié. En l'absence d'un tel système, il n'est nullement possible de déterminer de manière fiable et objective ni le nombre d'heures de travail réellement effectuées par le travailleur, ni leur répartition dans le temps. Dans ces conditions, il apparaît excessivement difficile pour les travailleurs de faire respecter les droits qui leur sont conférés par l'article 31, paragraphe 2, de la Charte des droits fondamentaux de l'UE et par la Directive 2003/88.

En outre, la réglementation relative au registre du temps de travail va dans le sens des conclusions rendues le 31 janvier 2019 par l'avocat général de la CJUE. Selon lui, les dispositions communautaires doivent être interprétées comme imposant aux entreprises l'obligation d'instaurer un système de mesure du temps de travail journalier effectif pour les travailleurs à temps plein qui n'ont pas expressément accepté, individuellement ou collectivement, d'effectuer des heures supplémentaires, et qui ne sont pas des travailleurs mobiles, de la marine marchande ou du secteur ferroviaire.

\section{II - LE TEMPS DE TRAVAIL}

La législation européenne ${ }^{1}$ définit le temps de travail effectif comme toute période durant laquelle le salarié est au travail, à la disposition de l'employeur, et dans l'exercice de

Directive 2003/88/CE, art. 2. 
son activité ou de ses fonctions. Elle est donc conçue par opposition à la période de repos, cette dernière étant entendue comme toute période qui n'est pas du temps de travail. Pour sa part, la législation nationale ${ }^{2}$ prévoit que le temps de travail correspond à la période durant laquelle le salarié est à son poste de travail. Mais cette définition, en apparence simple, cache une réalité plus complexe mise en exergue par l'obligation d'instaurer ce nouveau système d'enregistrement du temps de travail. Certaines décisions judiciaires récentes permettent de mieux analyser la situation.

La Directive définit le temps de travail comme "toute période durant laquelle le travailleur est au travail, à la disposition de l'employeur et dans l'exercice de son activité ou de ses fonctions, conformément aux législations ou pratiques nationales $»^{3}$. Selon la CJUE (arrêts Simap, Jaeger et Dellas), "la période de repos est définie, par opposition, comme «toute période qui n'est pas du temps de travail ». Le temps de travail est donc celui pendant lequel le travailleur est à la disposition de l'employeur, indépendamment de I'intensité du travail effectivement fourni ».

\section{III - LES CRITÈRES D'INTERPRÉTATION DE LA NOTION DE TEMPS DE TRAVAIL}

Les décisions prises par les tribunaux dans le cadre des affaires brièvement décrites ci-dessous sont conformes au cadre normatif et interprétatif actuel; elles visent à fournir une définition du temps de travail qui se veut exhaustive, et à faciliter la mise en œuvre du registre du temps de travail.

\section{A - LE TEMPS D'ASTREINTE}

Afin d'appréhender ces deux concepts, quatre décisions sont analysées ci-après : un arrêt de la Cour de Justice de I'UE (affaire Matzak) et trois arrêts de tribunaux espagnols ${ }^{5}$.

1- Selon la jurisprudence Matzak, il est exclu que les États membres maintiennent ou adoptent une définition moins restrictive de la notion de «temps de travail » que celle énoncée à l'article 2 de la Directive 2003/88. En effet, cet article doit être interprété en ce sens que le temps de garde qu'un travailleur passe à domicile avec l'obligation de répondre aux appels de son employeur dans un délai de 8 minutes, restreint très significativement les possibilités d'avoir d'autres activités.

2 - Les trois autres arrêts portent sur la distinction entre « temps de travail » et « temps de disponibilité » dans les situations de gardes inactives.

La décision $n^{\circ} 1249 / 2017 d u$ Tribunal Supremo du 19 novembre 2019 propose une synthèse permettant de formuler quelques principes généraux qui précisent les contours de ces deux notions :

- La notion de « temps de travail » est présumée dès lors que le salarié ne peut disposer librement de son temps, au lieu où il le souhaite;

- La présence dans les locaux de l'entreprise est largement déterminante pour la qualification de «temps de travail »;

- La notion de «temps de travail » est indépendante de l'intensité de l'activité ou de la nature productive de celle-ci;

2 Statut des Travailleurs, art. 34.

3 Décision du Tribunal Supremo n³0/2018 du 19 mars 2019.

4 CJUE, 21 février 2018, C-518/15.

5 Décision du Tribunal Supremo Catalogne n`3681/2018 du 17 octobre 2018. 
- Les déplacements sont comptabilisés comme du temps de travail ;

- Une distinction est faite entre le temps de travail productif et celui non productif, avec des incidences possibles sur la rémunération.

En ce qui concerne le "temps de disponibilité », selon la jurisprudence de la CJUE (résumée par la décision $n^{\circ} 225 / 2018$ du Tribunal Supremo de Madrid du 14 septembre 2018), s'il est consacré à une activité liée au travail, ce temps est de nature professionnelle, indépendamment de l'endroit où se trouve le salarié et sans possibilité pour les États membres de réinterpréter les notions de temps de travail et de périodes de repos définies par la Directive. L'impossibilité d'exercer d'autres activités est manifeste si, pendant la période de garde, le travailleur est physiquement présent sur le lieu déterminé par l'employeur.

\section{B - LE REGISTRE DU TEMPS DE TRAVAIL}

S'agissant du registre du temps de travail, la décision n654/2018 du Tribunal Supremo Canaries/Tenerife du 31 janvier 2019 est intéressante car, bien que le cadre réglementaire soit antérieur au Décret-loi royal 8/2019, elle renvoie à des aspects pertinents.

L'affaire concerne le salarié unique d'une entreprise qui prétend, sur la base de son propre témoignage et d'une capture d'écran de ses horaires sur le Web, que son temps de travail hebdomadaire est de 49 heures, et réclame le paiement correspondant aux heures supplémentaires effectuées. Après reconnaissance du bien-fondé de cette demande de règlement, l'entreprise en question a fait appel de la décision. Contrairement aux arguments avancés par l'entreprise, le Tribunal Supremo considère que l'absence de système d'enregistrement du temps de travail ne saurait favoriser l'employeur en termes d'exigences procédurales et faire peser sur les salariés l'obligation d'apporter la preuve des heures supplémentaires effectuées. L'entreprise doit donc mettre en place un système qui permette de mesurer le temps de travail journalier de chaque travailleur, et ainsi de prouver que les heures supplémentaires effectuées par ce dernier ont été rémunérées ou contrebalancées par des périodes équivalentes de repos compensateur. L'obligation d'imposer aux entreprises la mise en place d'un registre du temps de travail journalier de chaque travailleur met un terme définitif à ces controverses.

\section{C - LE TEMPS DE TRAVAIL}

De nombreux arrêts ont récemment défini le temps de travail, en tenant compte :

- de la participation volontaire à des événements de nature commerciale en dehors de la journée de travail, compensée par une période de repos ${ }^{6}$;

- des heures supplémentaires effectuées de 7h30 (ou 7h45) à 8h007 ;

- des déplacements effectués entre le domicile du salarié et celui du client (et vice-versa), dès lors que ces déplacements sont indispensables à l'activité de l'entreprise ${ }^{8}$;

- du temps de disponibilité obligeant les travailleurs à être prêts à intervenir auprès des clients en cas de besoin les samedis, dimanches et jours fériés, de $08 \mathrm{~h} 00$ à $20 \mathrm{h00}{ }^{9}$;

6 Décision du Tribunal Supremo n³0/2018 du 19 mars 2019.

7 Décision de l'Audiencia Nacional n¹47/2019 du 31 octobre 2019.

8 CJUE, 10 septembre 2015 (C266/14).

9 Décision du Tribunal Supremo Catalogne n³681/2018 du 17 octobre 2018. 


\section{ESPAGNE}

- du temps de chevauchement entre les équipes de garde successives, pendant lequel les infirmiers en fin de garde transmettent à ceux qui prennent la relève des informations sur le traitement donné à certains patients ou sur leur état de santé particulier ${ }^{10}$.

En revanche, ne sont pas qualifiés de «temps de travail »:

- le temps passé par le pompier officiant sur un aérodrome pour aller du bâtiment principal à celui où a lieu la relève. Durant ce laps de temps, le pompier n'est pas considéré comme étant à la disposition de l'employeur, ni en mesure d'exercer ses fonctions telles que définies dans la Convention et la jurisprudence de l'UE. L'argument à l'appui consiste à dire qu'il s'agit d'une « phase préliminaire, et non productive, analogue à celle du passage du vestiaire d'une entreprise au lieu de travail $»^{11}$;

- le temps consacré à la formation ${ }^{12}$, dans le cadre de l'offre de formation proposée par l'entreprise sans caractère obligatoire, sans lien direct avec des emplois spécifiques et sans que l'acceptation ou le refus de celle-ci ne porte à conséquence ;

- le temps passé sur l'île [des Baléares] par un travailleur embauché comme surveillant des Aires Marines, une fois la journée de travail terminée. II ressort de l'arrêt rendu ${ }^{13}$ que "la disponibilité téléphonique n'équivaut pas à du temps de travail ", étant donné $q^{\prime}$ '« une fois la journée de travail terminée, la présence sur l'île n'est pas imposée par l'entreprise mais découle de la nature du travail effectué »;

- le temps durant lequel le salarié doit être joignable, si aucun autre élément factuel ne prouve l'obligation pour celui-ci d'être également présent physiquement dans un lieu déterminé par l'employeur ${ }^{14}$;

- le temps durant lequel le travailleur est simplement disponible et joignable, sur le téléphone fourni par l'entreprise, sans qu'une durée minimum de disponibilité ait été fixée et sans devoir être présent physiquement dans un lieu déterminé ${ }^{15}$;

- la pause ou le repos de 30 à 45 minutes d'un conducteur de bus, car cela est expressément exclu par la Directive 2002/15/CE du 11 mars 2002 relative à l'aménagement du temps de travail des personnes exécutant des activités mobiles de transport routier ${ }^{16}$. Si, pendant la période de repos, un appel est reçu signalant un incident urgent, le travailleur peut refuser de donner suite à la demande.

10 Décision du Tribunal Supremo Andalousie\Séville n981/2018 du 20 décembre 2018.

11 Décision du Tribunal Supremo n¹249/2017 du 19 novembre 2019.

12 Décisions du Tribunal Supremo n²10/2017 du 20 février et n²3/2018 du 6 mars 2019.

13 Décision du Tribunal Supremo îles Baléares n9/2019 du 24 mai 2019.

14 Décisions du Tribunal Supremo Galice n662/2018 du 27 avril 2018 et n678/2018 du 8 mai 2018.

15 Décision de l'Audiencia Nacional n¹25/2018 du 20 septembre 2018.

16 Décision du Tribunal Supremo Madrid n²25/2018 du 14 septembre 2018. 